\title{
La eco-innovación en las empresas mexicanas de alojamiento turístico
}

\section{Eco-innovation in Mexican tourist accommodation companies}

\begin{abstract}
Dra. Marta Magadán Díaz es profesora e investigadora de la Facultad de Empresa y Comunicación de la Universidad Internacional de La Rioja (UNIR), España y directora de la editorial universitaria Septem Ediciones (marta.magadan@unir.net) (http:// orcid.org/0000-0003-3178-3215)
\end{abstract}

Dr. Jesús Israel Rivas García es profesor e investigador de la Facultad de Empresa y Comunicación de la Universidad Internacional de La Rioja (UNIR), España y director de marketing y estrategia de la editorial universitaria Septem Ediciones (jesus. rivas@unir.net) (http://orcid.org/0000-0003-0576-5961)

\section{Resumen}

La eco-innovación se muestra como uno de los grandes desafíos de la industria turística dada la cada vez mayor conexión entre calidad medioambiental y la buena marcha del sector. Si bien la literatura turística ha considerado el tema de la sostenibilidad del negocio en términos más amplios, la noción de eco-innovaciones representa un nuevo campo de investigación con estudios que aún investigan a fondo la adopción de eco-innovaciones entre las empresas turísticas. En este trabajo se aborda la evolución conceptual de la eco-innovación para, posteriormente desarrollar un marco analítico que explore tentativamente dicho concepto y su implantación en las empresas mexicanas de alojamiento turístico a través de dos características internas básicas de dichas organizaciones: sus resultados empresariales y sus respectivos tamaños, medidos en número de cuartos. La vía metodológica empleada ha sido el método del caso, realizando un conjunto de estudios de casos relativos a seis grupos hoteleros de capital netamente mexicano a través de evidencias documentales y entrevistas estructuradas. Las conclusiones derivadas de los hallazgos obtenidos sugieren, en primer lugar, que la eco-innovación está vinculada directamente con la evolución de los resultados empresariales y con el tamaño de las organizaciones y, en segundo lugar, que la eco-innovación se percibe como una apuesta estratégica generadora de ventajas comparativas para empresas mexicanas estudiadas independientemente de las señales emitidas por una demanda concienciada ecológicamente y más allá de las acciones reguladoras gubernamentales.

\begin{abstract}
Eco-innovation is shown as one of the great challenges of the tourism industry given the growing connection between environmental quality and its good performance. Although the tourism literature has considered the topic of business sustainability in broader terms, the notion of eco-innovations represents a new field of research with studies that still investigate in depth the adoption of eco-innovations among tourism companies. This work addresses the conceptual evolution of eco-innovation to subsequently develop an analytical framework that tentatively explores this concept and its implementation in Mexican tourist accommodation companies through two basic internal characteristics of these organizations: their business performances and their respective sizes, measured in number of rooms. The methodology used has been the case method, making a set of case studies related to six hotel groups of purely Mexican capital through documentary evidence and structured interviews. The conclusions derived from the findings suggest, firstly, that eco-innovation is directly linked to the evolution of business results and the size of organizations and, secondly, that eco-innovation is perceived as a strategic bet generating comparative advantages for Mexican companies studied, independently of the signals issued by an ecologically aware demand and beyond the governmental regulatory actions.
\end{abstract}

\section{Palabras clave I keywords}

Innovación, eco-innovación, sostenibilidad ambiental, gestión de empresas hoteleras, método de estudio de caso Sustainable tourism, environmental sustainability, business management, organizational change, enterprises, technological change.

Forma sugerida de citar: Magadán Díaz, M. \& Rivas García, J. I. (2018). La eco-innovación en las empresas mexicanas de alojamiento turístico. Retos Revista de Ciencias de la Administración y Economía, 15(8), 19-33. https://doi.org/10.17163/ret.n15.2018.02 


\section{Introducción}

La capacidad para competir de las organizaciones empresariales se ve, cada vez más, influida por su relación con la sostenibilidad y la innovación, bien actuando ambas por separado (Hitchens et al., 2005) o bien de forma sinérgica (Esty \& Winston, 2009), generando nuevos mercados para los productos ambientalmente benignos (Beise \& Rennings, 2005) y un nuevo campo de estudio académico, la eco-innovación (Fussler \& James, 1996; Jänicke, 2008). El binomio empresa-medio ambiente está transformando procesos, productos, mercados y estrategias comerciales al incorporar en la dirección estratégica empresarial los principios de sostenibilidad (Bengochea et al., 2006; Segarra et al., 2011). Por otra parte, la industria turística no es ajena a las nuevas tecnologías y a su impacto sobre las alteraciones en los patrones de conducta y pautas de consumo del turista actual (Agarwal, 2002; Aguiló et al., 2005). En suma, la eco-innovación -o innovación ambiental- podría tener un papel cada vez más revelador en la conformación de un modelo turístico adaptado a los principios de sostenibilidad ambiental (Hunter \& Shaw, 2007).

Las empresas del sector turístico están participando cada vez más en prácticas y medidas innovadoras, no solo para sostener el crecimiento, sino también como respuesta a la escala cambiante y la naturaleza del cambio ambiental global (Bell \& Ruhanen, 2016). Para algunas empresas, esto ha incluido el desarrollo y la adopción de innovaciones ecológicas (Bell \& Ruhanen, 2016). Si bien la literatura turística ha considerado el tema de la sostenibilidad del negocio en términos más amplios, la noción de eco-innovaciones representa un nuevo campo de investigación con estudios que aún investigan a fondo la adopción de estas estrategias entre las empresas turísticas (Tzschentke et al., 2008).

La eco-innovación, como concepto manejado en la literatura académica, es relativamente de reciente data (Peiró et al. , 2014), en el que encuentran espacio procedimientos organizacionales vinculados a la incorporación de aspectos medioambientales en diferentes puntos de la cadena de valor e, incluso, en sus mismos diseños y estrategias organizativas (Klewitz \& Hansen, 2014). Pero su interés ha crecido de modo relevante como consecuencia -directa o indirecta- de las nuevas opciones y posibilidades de negocio que se abren con la eco-innovación y que, especialmente, podrían despertar un interés especial en las pequeñas y medianas empresas de cara a mejorar de su eficiencia y competitividad: i) con la implementación de nuevos o mejorados procesos y productos, o ii) con una reconsideración profunda de sus modelos de negocio. La eco-innovación ofrece, como se indicó anteriormente, un camino de eficiencia competitiva capaz de abrir nuevos mercados (Ruiz et al., 2013). Aunque la eco-innovación se cimente sobre la base del progreso técnico (Velázquez \& Vargas, 2015), puede, así mismo, impulsarse sencillamente a través de argumentos que manifiesten una genuina preocupación por el entorno o por una evidente necesidad económica en la organización empresarial, capaz de ver las ganancias de eficiencia y competitividad a las que ya se ha hecho referencia. En cualquier caso, la característica singular de un "proceso eco-innovador» en las organizaciones es que ha de promover y consustanciarse siempre en una clara ganancia para el medio ambiente.

La eco-innovación se muestra como uno de los grandes desafíos de la industria turística dada la cada vez mayor conexión entre calidad medioambiental y la buena 
marcha del sector (Tzschentke et al., 2008). De hecho, numerosos estudios sobre la industria hotelera han demostrado que el compromiso con las prácticas ambientales mejora el desempeño financiero de los hoteles (Tarí et al., 2010; Tzschentke et al., 2008). A pesar del progreso de la industria para ser sostenible, los hoteles todavía luchan con la forma más efectiva de promover su estado verde (Peiró et al., 2014).

\section{Revisión de la literatura}

\subsection{Concepto de eco-innovación}

El término eco-innovación se puede definir de muy diversas formas (ver tabla 1), pero podemos señalar por su amplitud y generalidad, al menos, dos definiciones que tratan la eco-innovación como: i) un nuevo elenco de procesos y productos capaz de, por un lado, incrementar el valor para clientes y empresas y, por otro, de favorecer una reducción significativa de las externalidades negativas sobre el medio (Fussler \& James, 1996; Mossalanejad, 2011); ii) la aparición de cualquier novedad o mejora en productos o procesos, rediseño organizacional o nuevas posibilidades de marketing que, conjunta o aisladamente, sean capaces de optimizar el uso de los recursos naturales minimizando los impactos negativos asociados a tales cambios (Alonso et al., 2016).

Las innovaciones ecológicas no están exclusivamente relacionadas con las implementaciones individuales de las empresas, sino que ocupan un lugar muy importante en los modelos comerciales ecológicos. Esto no solo crea productos respetuosos con el medio ambiente, sino que también genera una economía más sostenible. Finalmente, la eco-innovación puede contribuir al establecimiento de redes empresariales y sociales, aumento del capital social, cooperación empresarial y creación de nuevas relaciones entre el sector público y el privado (Alonso et al., 2016). Por estos motivos, todas las definiciones de eco-innovación concuerdan con el valor creciente del medio ambiente y reflejan dos efectos de las eco-innovaciones: disminución significativa de externalidades negativas y mejor aprovechamiento de los factores productivos obtenidos del medio ambiente (Hojnik \& Ruzzier, 2016).

Tabla 1. Definiciones de eco-innovación

\begin{tabular}{|l|l|}
\hline \multicolumn{1}{|c|}{ Autor } & \multicolumn{1}{c|}{ Concepto de eco-innovación } \\
\hline (Fussler \& James, 1996) & $\begin{array}{l}\text { Nuevos productos y procesos que ofrecen valor al cliente y al ne- } \\
\text { gocio, pero que disminuyen significativamente los impactos am- } \\
\text { bientales. }\end{array}$ \\
\hline (Klemmer et al., 1999) & $\begin{array}{l}\text { Todas aquellas medidas de los actores sociales relevantes (empre- } \\
\text { sas, políticos, sindicatos, asociaciones, iglesias, hogares particu- } \\
\text { lares) que: i) desarrollan nuevas ideas, comportamientos, produc- } \\
\text { tos y procesos, ii) los aplican o los introducen, y iii) contribuyen } \\
\text { a la reducción de las cargas ambientales o a lograr objetivos de } \\
\text { sostenibilidad especificados ecológicamente. }\end{array}$ \\
\hline (Andersen, 2002) & Toda innovación capaz de atraer rentas verdes al mercado. \\
\hline
\end{tabular}




\begin{tabular}{|l|l|}
\hline (Little, 2005) & $\begin{array}{l}\text { La creación de nuevos espacios de mercado, productos, servicios } \\
\text { o procesos impulsados por problemas sociales, ambientales o de } \\
\text { sostenibilidad. }\end{array}$ \\
\hline (Charter \& Clark, 2007) & $\begin{array}{l}\text { Proceso en el que las consideraciones de sostenibilidad (am- } \\
\text { bientales, sociales, financieras) se integran en los sistemas de la } \\
\text { empresa, desde la generación de ideas hasta la investigación y } \\
\text { el desarrollo (I+D) y la comercialización. Se aplica a productos, } \\
\text { servicios y tecnologías, así como a nuevos modelos comerciales } \\
\text { y de organización. }\end{array}$ \\
\hline $\begin{array}{l}\text { (Kemp \& Arundel, 1998) } \\
\text { (Rennings, 2000) } \\
\text { (Kennings \& Zwick, 2003) } 2010)\end{array}$ & $\begin{array}{l}\text { La producción, asimilación o explotación de un producto, un } \\
\text { servicio, un proceso de producción, un método comercial o de } \\
\text { gestión novedoso para la organización (que lo desarrolla o adop- } \\
\text { ta) y que favorece, a lo largo de su ciclo de vida, una reducción } \\
\text { del riesgo ambiental, contaminación y otros impactos negativos } \\
\text { derivados del uso de los recursos (incluido el uso de energía) en } \\
\text { comparación con las alternativas relevantes. }\end{array}$ \\
\hline (Oltra \& Saint Jean, 2009) & $\begin{array}{l}\text { Procesos, prácticas, sistemas y productos nuevos o modificados } \\
\text { que benefician al medio ambiente y contribuyen a la sostenibili- } \\
\text { dad ambiental }\end{array}$ \\
\hline (Freeman, 1996) & $\begin{array}{l}\text { Es lo mismo que otros tipos de innovación, pero con dos distin- } \\
\text { ciones importantes: i) representa una innovación que da como } \\
\text { resultado una reducción del impacto ambiental y ii) su alcance } \\
\text { puede ir más allá de los límites organizacionales convenciona- } \\
\text { les de la organización innovadora e involucrar pactos o acuerdos } \\
\text { sociales más amplios que desencadenan cambios en las normas } \\
\text { socioculturales y estructuras institucionales existentes. }\end{array}$ \\
\hline
\end{tabular}

De los diferentes conceptos de eco-innovación es posible observar que ésta se manifestaría como un proceso tridimensional, al nutrirse de la Ecología, la Economía y la Tecnología (Hong \& Shuai, 2008; van Berkel, 2007). Toda organización se situaría en ese espacio tridimensional buscando progresar, considerando los costes de oportunidad asociados a un avance sin mejoras ambientales que pudieran haber redundado en mejores resultados empresariales o, por ejemplo, haber eludido las consecuencias administrativas (esquema sancionador conforme al polluter pays principle) y sociales (escasez de recursos y empobrecimiento de las generaciones futuras) derivadas un avance a espaldas del medio ambiente.

Por lo tanto, la eco-innovación busca equilibrar el desarrollo comercial y la productividad con las preocupaciones ecológicas y el respeto por el medio ambiente, de modo que el sector turístico pueda conservar y utilizar eficientemente los recursos naturales. Como resultado, la eco-innovación influye directamente en el crecimiento económico y social a través del cuidado del medio ambiente, el mantenimiento y la creación de nuevos empleos y la adquisición de nuevas habilidades, nuevos productos y nuevos negocios, todos los cuales influyen positivamente en la economía (Alonso et al., 2016). 
Todavía no hay un consenso sobre la definición del concepto de eco-innovación, lo que implica que nos encontramos en una etapa inicial de construcción y desarrollo en este campo de investigación, que es aún más patente cuando el análisis se centra en el sector turístico, dada la falta de estudios relacionados con este tema (García et al., 2015). De hecho, al aproximarse a los pocos estudios existentes, se puede observar que el concepto de eco-innovación predominante en el análisis de los alojamientos hoteleros se percibe como un binomio entre gestión ambiental e innovación, orientado a la mejora continua de la organización (Velázquez \& Vargas, 2015).

\subsection{Evolución sectorial de los estudios sobre eco-innovación}

La investigación previa sobre sostenibilidad en el sector turístico se originó hace dos décadas como un análisis de la adopción de prácticas amigables con el medio ambiente entre otras obras (Alonso et al., 2015) y su impacto económico (Perramon et al., 2014). La sostenibilidad afecta a la economía de tres maneras: i) tiene un efecto directo a través del ahorro de energía; iii) tiene un efecto indirecto porque una imagen respetuosa con el medio ambiente crea una ventaja competitiva; y iii) el negocio gana la ventaja de posicionarse como una empresa ecológica (Best \& Thapa, 2013; Hsiao \& Chuang, 2016). En menor medida, investigaciones anteriores han encontrado que hay un impacto social (Bagur et al., 2013) y un impacto en la adquisición de capacidades únicas (Tesone, 2004).

Sin embargo, la investigación en eco-innovación es más reciente; por lo tanto, se encuentra en una etapa inicial o embrionaria (Alonso et al., 2016). Como es el caso en otros campos de estudio, comenzó en el sector manufacturero (Segarra et al., 2014). En particular, la creación y adopción de innovaciones ecológicas se han analizado en este sector, y se han identificado una serie de factores desencadenantes para su desarrollo (Alonso et al., 2016): i) la inversión en Investigación y Desarrollo e Innovación (I+D+i) y la capacidad de exportación de una innovación (Horbach et al., 2012), tienen poco lugar en el sector de los servicios; ii) la posesión o el tamaño de una masa crítica de recursos y capacidades, el dominio de ciertas capacidades tecnológicas y la existencia de características organizacionales específicas para cada empresa (Biondi et al., 2002), pueden pertenecer a todo tipo de sectores. En cualquier caso, parece que existe un consenso que indica que la regulación ambiental y los mecanismos del mercado son los principales desencadenantes para la creación, el desarrollo y la difusión de las eco-innovaciones (Horbach et al., 2012); iii) Proviene del entorno en el que la compañía desarrolla su actividad que de la propia empresa (del Río, 2005). Por lo tanto, investigaciones posteriores señalaron que los factores internos podrían impulsar más que los externos porque las empresas tienen poco o ningún control sobre los factores externos (Bossle et al., 2016).

También se han identificado barreras para la implementación de eco-innovaciones en el sector de servicios (Alonso et al., 2016), incluyendo la falta de capacidad de absorción en los procesos, recursos humanos capacitados, madurez comercial y capacidades tecnológicas.

La eco-innovación en el sector turístico ha sido poco documentada en investigaciones académicas o artículos científicos (Alonso et al., 2016). El vínculo turismo-medio ambiente se pone de manifiesto, al menos en tres aspectos: i) el medio ambiente es 
la base de desarrollo de muchos atractivos turísticos (turismo de naturaleza, turismo activo, turismo rural, turismo cinegético, etc.); ii) el medio ambiente es la fuente de extracción de los factores productivos empleados en toda organización; y iii) el medio ambiente es el vertedero al que van a parar los residuos y emisiones contaminantes. Los temas que se han estudiado en la literatura académica incluyen el papel de las eco-innovaciones en la elección de los establecimientos turísticos, como los restaurantes (Teng \& Chang, 2014), los tipos de eco-innovaciones adoptadas por los restaurantes (Daim et al., 2013; Rodgers, 2007) y por los hoteles (Bastič \& Gojčič, 2012; Horng et al., 2016), y la importancia de utilizar eco-innovaciones en el diseño del entorno físico de los restaurantes para generar una atmósfera innovadora (Horng et al., 2013).

Es importante destacar que las eco-innovaciones incluyen numerosas actividades que afectan a las empresas y los destinos turísticos en áreas como la energía; reciclaje; agua; desarrollo de nueva construcción; diseño de interiores; proyectos de ingeniería; respuestas a la degradación ambiental externa; nuevos productos, procesos y modelos comerciales; adaptaciones de productos y materiales existentes; nuevos materiales; el uso de productos eco-biológicos; la ordenación del territorio; y la industria del bienestar, que, en algunas regiones, también puede incluir el desarrollo y la transformación de productos orgánicos, ecoturismo y turismo terapéutico (Alonso et al., 2016). Si consideramos que cada eco-innovación implica la combinación de factores con numerosas actividades, podemos concluir que la generación, el desarrollo, la adopción y la difusión de las eco-innovaciones son diferentes y mucho más complejas que las de otras innovaciones (Karakaya et al., 2014) porque cada eco-innovación comprende varias innovaciones (Alonso et al., 2016; Velázquez \& Vargas, 2015).

\subsection{Planteamiento de hipótesis}

Inicialmente los propulsores de la eco-innovación podrían situarse tanto en el lado de la demanda -mercado- como en el lado de la oferta -capacidad de la organización-. Así, se pueden citar como impulsores de la misma: i) las exigencias por parte de los clientes, ii) las regulaciones existentes en el mercado que la promueven o imponen, iii) la mejora de la reputación de la organización, iv) la reducción de costes o v) el compromiso empresarial con la sociedad, en términos de responsabilidad social corporativa.

En este estudio se realizará una tentativa de aproximación correlacional a la relación entre eco-innovación y dos hipótesis vinculadas con las características internas de la empresa alojativa: su resultado empresarial (medido en términos de facturación anual) y su tamaño (medido en relación a su capacidad alojativa).

Existe una relación directa entre rendimiento financiero, resultados empresariales y eco-innovación que ha sido puesta de manifiesto en diversos trabajos (Doran \& Ryan, 2012; Przychodzen \& Przychodzen, 2015): una buena solidez financiera es una condición necesaria para el desarrollo de inversiones en eco-innovación y, al tiempo, la generación de resultados positivos a lo largo del tiempo, inclina a las empresas alojativas a realizar una apuesta por la eco-innovación. Además, esa relación tiende a ser biunívoca: empresas alojativas con buenos resultados se inclinan por la eco-innovación y empresas alojativas eco-innovadoras tienden a obtener mejores resultados que las no eco-innovadoras. A partir de lo indicado anteriormente, se propone la siguiente hipótesis: 
H1: Cuanto mayor es la facturación de la empresa alojativa, mayor es el incentivo a eco-innovar

Las organizaciones hoteleras no evolucionan hacia la eco-innovación desde posiciones similares. Un factor que puede ser decisivo es el tamaño (De-Marchi \& Grandinetti, 2012; Chen, 2008, Demirel \& Kesidou, 2011), al lograr incentivar y agilizar los procesos de eco-innovación. Existen trabajos que señalan la alta correlación entre tamaño y eco-innovación, y en ellos se sostiene que las organizaciones de mayor dimensión tienden a ser más proclives a apostar por la eco-innovación (Bowen, 2000; De Marchi \& Grandinetti, 2012), lo que puede verse como una consecuencia asociada a las mayores presiones gubernamentales recibidas y a un mayor control en el cumplimiento de la normativa ambiental.

Por otra parte, del análisis interno de las organizaciones empresariales (Churchill \& Levis, 1983; Greiner, 1997), la cuestión planteada es si el tamaño condiciona positiva o negativamente la apuesta por la eco-innovación (Molero \& García, 2008; Lazaric \& Dennis, 2005; Brunnermeier \& Cohen, 2003). A partir de lo indicado anteriormente, se propone la segunda hipótesis:

H2: Cuanto mayor es el número de habitaciones de la empresa alojativa, mayor es el incentivo a eco-innovar

\section{Metodología y muestra}

La metodología empleada para realizar el estudio empírico es el método del caso, porque se considera que se cumplen dos condiciones o dimensiones básicas (Yin, 2009): el tipo de preguntas que se busca responder con el desarrollo de esta investigación y, finalmente, que el problema abordado es actual.

Creswell (2005) considera que en un estudio de casos debe haber un control cruzado de fuentes de datos. A través de ella, aseguramos la validez constructiva, ya que la utilización de diferentes fuentes de datos y métodos de análisis nos permitirá conseguir una imagen más precisa de la realidad que estamos investigando (Johnston et al., 1999).

En esta investigación se han utilizado distintos métodos de recogida de información: a) evidencia documental, que pudo obtenerse a través de la publicidad, catálogos, informes y memorias de las empresas, así como datos financieros, entre otros y b) la realización de entrevistas estructuradas vía Skype y e-mail.

Las empresas alojativas seleccionadas (muestreo por conveniencia) son seis cadenas hoteleras pertenecientes a los principales grupos hoteleros nacionales mexicanos, que han sido nombradas como A1, A2, A3, A4, A5 y A6. Seguidamente (ver tabla 2) se muestran de modo resumido el perfil de cada una de las organizaciones estudiadas.

\section{Análisis de los resultados}

El sector turístico, en general, y el hotelero, en particular, como tantos otros sectores, no ha sido ajeno a los cambios de actitud política y social en relación al medio ambiente y, su apuesta por la sostenibilidad ambiental. En este nuevo contexto, las empresas alojativas han tenido que hacer frente a los nuevos retos planteados por aquellas innovaciones ambientales que, incardinadas en sus respectivos procesos internos, son capaces de ofre- 
cer una vía de compatibilidad entre la actividad empresarial propia de los hoteles y el medio ambiente. Sin embargo, dichas eco-innovaciones conllevan, además de una concienciación sobre la necesidad de protección y uso equilibrado (sostenible) del medio, una apuesta financiera cuyos retornos tienden a visualizarse no tanto en el corto como en el medio o largo plazo. Eso conduce, en muchos casos, a que las empresas deban repensar su estructura organizativa para obtener ventajas competitivas construidas sobre este subgrupo de innovaciones de naturaleza ecológica.

Como primera hipótesis se plantea que cuanto mayor es la facturación de la empresa alojativa, mayor es el incentivo a eco-innovar.

Recurriendo a la información obtenida a través del análisis de casos, encontramos que las organizaciones van tomando mayor conciencia ambiental. El representante de A1 reconoce que fue en los últimos años cuando la organización comenzó a tomarse en serio el cuidar la relación entre sus hoteles y el medio. Señala que comenzaron primeramente con un acercamiento a la idea de sostenibilidad desde las personas para, posteriormente, avanzar hacia el entorno. Su organización detectó el interés creciente por parte de su clientela en llevar a cabo un ocio respetuoso con personas, culturas y medio a lo que se añadió la preocupación de las administraciones gubernamentales que se fue materializando en normativas de obligado cumplimiento. Asumen haber llegado a esa conciencia y sensibilidad ambiental empujados por la demanda. Los representantes entrevistados de A4, A5 y A6 ofrecen un argumentario similar: actúan en términos de sensibilidad ambiental (certificaciones y planes de sostenibilidad ambiental, equipamientos eficientes energéticamente, etc.) movido por la demanda y las exigencias gubernamentales.

En todos estos casos, se subraya que la capacidad financiera de las respectivas organizaciones posibilitó introducir distintos procedimientos de gestión ambiental orientados al logro de certificaciones y sellos. Con el tiempo, esa formalización e institucionalización de buenas prácticas ambientales dentro de cada organización se ha ido concretando en unos mejores resultados al lograr alcanzar un rango de clientela cada vez más concienciada con el respeto al entorno.

Los representantes de A2 y A3 exponen con entusiasmo los logros de sus respectivas organizaciones en materia de eco-innovación. Para ambos, más allá de ser o tratarse de una necesaria adaptación a los cambios sociales concretados en normas gubernamentales o una percepción de las nuevas inquietudes de su clientela, de lo que se trata es de avanzar en la mejora de la propia organización. Para estos interlocutores, apostar por la eco-innovación es tener visión de largo plazo: se ahorra energía y, por tanto, se reducen costos pero, además, se incrementa el prestigio de la propia organización. En A2 y A3, sostienen que su apuesta por la sostenibilidad ambiental a través de la adopción de innovaciones retroalimenta positivamente los resultados económicos de la organización. Para A2 y A3, la eco-innovación puede defenderse en sus organizaciones no solo por la necesidad de construir una conciencia de respeto con el medio, sino que la misma racionalidad económica a largo plazo hace ver en la adopción de innovaciones ambientales una oportunidad de distanciar ingresos y costes para hacer avanzar los beneficios. 


\begin{tabular}{|c|c|c|c|c|c|c|}
\hline 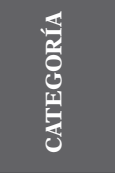 & 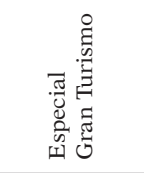 & 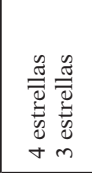 & 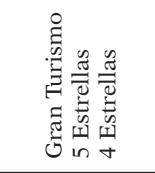 & 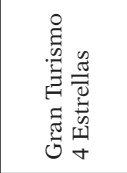 & 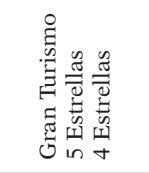 & 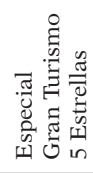 \\
\hline 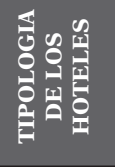 & 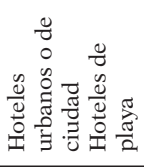 & 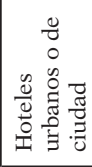 & 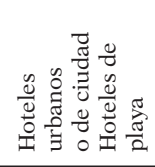 & 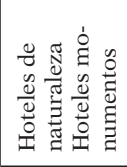 & 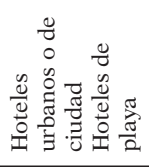 & $\begin{array}{l}\frac{0}{0} \\
0 \\
\frac{3}{0} \\
\frac{0}{0} \\
0 \\
0 \\
0\end{array}$ \\
\hline 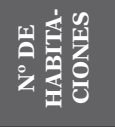 & $\begin{array}{l}\stackrel{\Xi}{\Xi} \\
\stackrel{+}{+}\end{array}$ & $\begin{array}{l}\stackrel{8}{ } \\
\infty \\
\dot{J}\end{array}$ & $\begin{array}{l}0 \\
\infty \\
0 \\
0 \\
0\end{array}$ & $\begin{array}{l}\stackrel{8}{0} \\
\text { in }\end{array}$ & $\begin{array}{l}8 \\
\stackrel{0}{0} \\
\text { in }\end{array}$ & $\underset{\sim}{\stackrel{0}{\circ}}$ \\
\hline 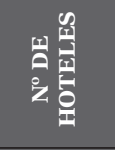 & in & $\stackrel{n}{m}$ & 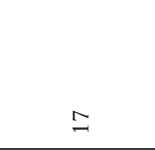 & $\bar{n}$ & $\tilde{N}$ & $\stackrel{\circ}{\circ}$ \\
\hline 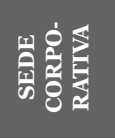 & 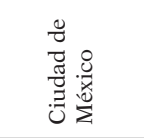 & 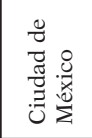 & 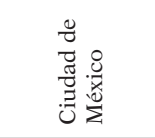 & 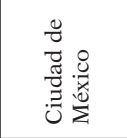 & 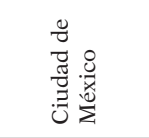 & 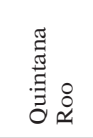 \\
\hline 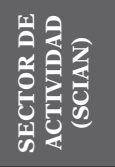 & $\begin{array}{l}\stackrel{\&}{\circ} \\
\underset{\infty}{1}\end{array}$ & $\underset{\underset{N}{\Xi}}{\underset{\Xi}{\Xi}}$ & $\underset{N}{\exists}$ & $\underset{N}{\equiv}$ & $\underset{N}{\exists}$ & $\underset{⿱ 亠}{\Xi}$ \\
\hline 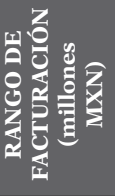 & 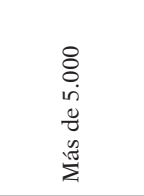 & 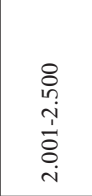 & & $\begin{array}{l}\stackrel{8}{0} \\
\stackrel{1}{1} \\
\stackrel{0}{1} \\
\end{array}$ & $\begin{array}{l}\stackrel{8}{0} \\
\stackrel{1}{1} \\
\stackrel{1}{8} \\
\stackrel{\sim}{1}\end{array}$ & $\begin{array}{l}\stackrel{8}{0} \\
\stackrel{1}{1} \\
\stackrel{1}{0} \\
\stackrel{4}{1}\end{array}$ \\
\hline 的是 & $\begin{array}{l}\stackrel{8}{\circ} \\
\stackrel{1}{\wedge}\end{array}$ & $\begin{array}{l}8 \\
0 \\
\frac{1}{1} \\
\frac{1}{0} \\
\text { in }\end{array}$ & \begin{tabular}{l}
8 \\
8 \\
1 \\
$\vdots$ \\
$\vdots$ \\
\hdashline
\end{tabular} & $\begin{array}{l}8 \\
\vdots \\
10 \\
\vdots \\
\vdots \\
0\end{array}$ & $\begin{array}{l}8 \\
8 \\
1 \\
\vdots \\
\vdots \\
0\end{array}$ & 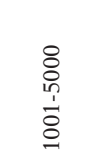 \\
\hline 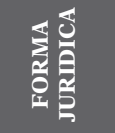 & $\begin{array}{l}己 \\
0 \\
0 \\
0 \\
\text { चे }\end{array}$ & $\begin{array}{l}己 \\
0 \\
0 \\
0 \\
\text { के }\end{array}$ & $\begin{array}{l}z \\
0 \\
0 \\
0 \\
\text { is }\end{array}$ & ஸే & $\begin{array}{l}8 \\
0 \\
o \\
\text { d }\end{array}$ & 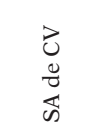 \\
\hline 45 & in & $\stackrel{2}{\sim}$ & $\stackrel{\vartheta}{-}$ & q & $\hat{n}$ & $\stackrel{m}{m}$ \\
\hline 牙艺 & $\bar{\psi}$ & $\stackrel{2}{2}$ & z & 安 & 要 & \& \\
\hline
\end{tabular}


Existe una relación directa entre rendimiento financiero, resultados empresariales y eco-innovación (Doran \& Ryan, 2012; Przychodzen \& Przychodzen, 2015): una buena solidez financiera es una condición necesaria para el desarrollo de inversiones en eco-innovación y, al tiempo, la generación de resultados positivos a lo largo del tiempo, inclina a las empresas alojativas a realizar una apuesta por la eco-innovación. Podemos derivar el siguiente resultado sobre la base de las observaciones efectuadas:

Resultado 1. Las empresas alojativas con mayor facturación tienen más incentivos a eco-innovar porque perciben las eco-innovaciones como inversiones cuyos retornos a medio y largo plazo les permiten consolidar una ventaja competitiva derivada de la valoración positiva por parte de una clientela cada vez más sensible a su relación con el medio.

Como segunda hipótesis planteamos que cuanto mayor es el número de habitaciones de la empresa alojativa, mayor es el incentivo a eco-innovar.

Recurriendo a la información obtenida a través del análisis de casos, encontramos que todos los responsables de los grupos analizados reconocen la necesidad de una mejor gestión de residuos, uso óptimo de la energía y el agua en todos los hoteles de sus respectivas organizaciones. Muy especialmente, en los casos de A1, A2 y A3, se subraya la relación directa entre esta preocupación directiva y el tamaño del hotel, especialmente por el control de costes indirectos variables asociados a los procesos de consumo y operaciones dentro de las unidades alojativas. A2 y A3 descartan trasladar la carga de la conciencia al cliente porque animarlo, por ejemplo, a usar el agua con responsabilidad es una llamada de atención a su conciencia, pero no es una solución empresarial adecuada a los desafíos que se plantean en la relación alojamiento turístico y entorno.

Por tanto, el tamaño de las empresas alojativas, medido en número de cuartos pueden incentivar y agilizar los procesos de eco-innovación (De Marchi \& Grandinetti, 2012; Chen, 2008, Demirel \& Kesidou, 2011). Las grandes empresas son más proclives a realizar eco-innovación (Bowen, 2000; De Marchi \& Grandinetti, 2012). Esto es debido a que soportan mayores presiones gubernamentales y están más vigiladas por la Administración gubernamental.

Por otra parte, al analizar la estructura interna de la empresa y, teniendo presente que esta estará relacionada con su evolución (Nelson, 1982; Churchill \& Levis, 1983; Greiner, 1997), así como con su tamaño, la pregunta de investigación que se propone es si esta variable puede suponer un freno a la eco-innovación (Molero \& García, 2008; Lazaric \& Dennis, 2005; Brunnermeier \& Cohen, 2003). Podemos derivar el siguiente resultado sobre la base de las observaciones efectuadas:

Resultado 2. Cuanto mayor es el número de cuartos en los establecimientos hoteleros mayor es su incentivo a eco-innovar de cara a realizar una gestión eficiente de los recursos disponibles y obtener así reducciones significativas de costos variables indirectos.

\section{Conclusión y discusión}

A partir de los resultados obtenidos se ha podido confirmar que en las empresas hoteleras mexicanas analizadas: i) cuanto mayor es la facturación de la empresa alojativa, mayor es el incentivo que tiene para eco-innovar, y ii) cuanto mayor es el número de 
habitaciones, mayor es el incentivo a eco-innovar. Sin embargo, los argumentarios esgrimidos permiten clasificar a estas organizaciones en dos grupos: i) eco-innovadoras reactivas (sus informes anuales recogen los avances en materia ambiental, certificando progresivamente sus hoteles en calidad ambiental y subrayando el cambio de tendencia del mercado que cada vez más se orienta a productos y servicios sostenibles) y ii) eco-innovadoras proactivas o anticipativas (desarrolla informes anuales de sostenibilidad con las actuaciones llevadas a cabo en este campo procurando ir por delante de las normas en esta materia y tratando de hacer uso de eco-innovaciones en sus alojamientos).

En el primer grupo (A1, A4, A5 y A6) el "push factor" fue doble: primeramente, la normativa reguladora gubernamental a cumplir y, en segundo lugar, la mayor sensibilidad y preocupación por el entorno de los clientes. A partir de ahí, toman estas organizaciones conciencia de que eco-innovar no es una obligación costosa sino una estrategia de largo plazo para reducir costes y mejorar la posición de las diferentes empresas de sus respectivas organizaciones. En el segundo grupo (A2 y A3), hay una cierta anticipación y visión «más allá» de las exigencias y demandas del mercado que hace que tomen el control de los procesos y operaciones internas de las unidades alojativas de sus respectivos grupos, orientándolas hacia la eco-innovación desde la propia convicción que esa es la estrategia adecuada para hacer una hotelería de calidad y rentable.

Las organizaciones empresariales buscan amoldarse a las condiciones derivadas de un nuevo marco socioeconómico y ecológico y sus líderes están empezando a aceptar el valor de una cultura organizacional capaz de transformar y orientar a las empresas hacia el desarrollo sostenible. La primera conclusión es ver en la eco-innovación como una fuente de ventajas competitivas (Buhl et al., 2016; Kemp \& Horbach, 2007; Rosen, 2001) en el medio y largo plazo asociadas a: i) una reducción significativa de costes, ii) una mejora de la reputación empresarial, iii) un acceso a segmentos del mercado (targets) que tienen 'conciencia ecológica' y desean disfrutar de su ocio de un modo sostenible (respetuoso) con el entorno.

La segunda conclusión es subrayar el papel impulsor de las políticas gubernamentales para orientar a las empresas hoteleras hacia unas buenas prácticas ambientales. De hecho, las eco-innovaciones y su implementación empresarial en el sector alojativo deberían ser apoyadas por los diferentes instrumentos o mecanismos (incentivos, subvenciones y ventajas fiscales, principalmente) y políticas gubernamentales disponibles (políticas de difusión de información, de transferencia de tecnología y de creación de asociaciones y clusters de naturaleza mixta público-privada) con el objeto de minimizar las posibles barreras del conocimiento (Doran \& Ryan, 2012; Hojnik \& Ruzzier, 2016). En unos casos, esa orientación tendrá una naturaleza reactiva: la normatividad actúa definiendo los límites de lo admisible ambientalmente (Horbach et al., 2012; Rennings, 2000). En otros, puede abrirse un diálogo y una pedagogía social que, más allá de la relación gobierno-empresa alojativa, promueva ciudadanos con conciencia ecológica y cuyas decisiones en el mercado estén modeladas por aquella.

Este trabajo presenta importantes limitaciones vinculadas a la selección de la muestra que requerirán en el futuro un estudio más amplio y detallado para ver la evolución de la eco-innovación turística en un país tan rico como diverso en términos turísticos. El perfil de los grupos considerados está muy marcado por su carácter urbano pero sería deseable evaluar unidades alojativas con orientaciones diferentes 
para detectar si existen diferencias significativas en la incorporación de innovaciones ambientales dependiendo del mercado al que se dirigen las empresas.

En resumen, este estudio trata de ofrecer una modesta tentativa de aproximación a un tema de importancia capital para la gestión estratégica de las empresas hoteleras en general que en Europa avanza de modo destacado y que se intuye que seguirá un ritmo análogo en el tejido empresarial mexicano, considerado este, como uno de los más importantes de América Latina.

\section{Referencias}

Agarwal, S. (2002). Restructuring Seaside Tourism: The Resort Lifecycle. Annals of Tourism Research, 29, 25-55. DOI: 10.1016/S0160-7383(01)00002-0.

Aguiló, E., Alegre, J., \& Sard, M. (2005). The Persistence of the Sun and Sand Tourism Model. Tourism Management, 26, 219-231. DOI: 10.1016/j.tourman.2003.11.004.

Alonso, M., Bagur, L., Llach, J., \& Perramon, J. (2015). Sustainability in small tourist businesses: the link between initiatives and performance. Current Issues in Tourism, 8, 1-20. DOI: 10.1080/13683500.2015.1066764.

Alonso, M., Rocafort, A., \& Borrajo, F. (2016). Shedding light on eco-innovation in tourism: A critical analysis. Sustainability, 8(12), 1262-1274. DOI:10.3390/su8121262.

Andersen, M. M. (2002). Organising Interfirm Learning: as the Market Begins to Turn Green. In T. Bruijn y A. Tukker, Andersen, M.M. (2002) 'Organising Interfirm Learning: as the Market Begins to Turn Green', in de Bruijn, Partnership and Leadership. Building Alliances for a Sustainable Future (pp. 103-119). Dordrecht: Kluwer Academic Publishers.

Bagur, L., Llach, J., \& Alonso, M. (2013). Is the adoption of environmental practices a strategical decision for small service companies? An empirical approach. Management Decision, 51(1), 41-62. DOI: 10.1108/00251741311291300.

Bastič, M., \& Gojčič, S. (2012). Measurement scale for eco-component of hotel service quality. International Journal of Hospitality Management, 31(3), 1012-1020. DOI: 10.1016/j. ijhm.2011.12.007.

Beise, M., \& Rennings, K. (2005). Lead markets and regulation: a framework for analyzing the international diffusion of environmental innovations. Ecological Economics, 52(1), 5-17. DOI: 10.1016/j.ecolecon.2004.06.007.

Bell, C., \& Ruhanen, L. (2016). The diffusion and adoption of eco-innovations amongst tourism businesses: the role of the social system. Tourism Recreation Research, 41(3), 291-301. DOI: $10.1080 / 02508281.2016 .1207881$.

Bengochea, A., Magadán, M., \& Rivas, J. (2006). Actividad turística y medio ambiente [Tourist activity and environment]. Oviedo: Septem Ediciones.

Best, M. N., \& Thapa, B. (2013). Motives, facilitators and constraints of environmental management in the Caribbean accommodations sector. Journal of Cleaner Production, 52, 165175. DOI: $10.1016 /$ j.jclepro.2013.03.005.

Biondi, V., Iraldo, F. \& Meredith, S. (2002). Achieving sustainability through environmental innovation: the role of SMEs. International Journal of Technology Management, 24(5-6), 612-626. DOI: 10.1504/IJTM.2002.003074.

Bossle, M. B., de Barcellos, M., Vieira, L. \& Sauvée, L. (2016). The drivers for adoption of eco-innovation. Journal of Cleaner Production, 113, 861-872. DOI: 10.1016/j.jclepro.2015.11.033.

Bowen, F. E. (2000). Environmental visibility: A trigger of green organizational response? Business Strategy and the Environment, 9(2), 92-107.

Brunnermeier, S., \& Cohen, M. (2003). Determinants of environmental innovation in US manufacturing industries. Journal of Environmental Economics and Management, 45, 278-293. DOI: 10.1016/S0095-0696(02)00058-X. 
Buhl, A., Blazejewski, S., \& Dittmer, F. (2016). The more, the merrier: Why and how employee-driven eco-innovation enhances environmental and competitive advantage. Sustainability, 8(9), 946. DOI: 10.3390/su8090946.

Charter, M., \& Clark, T. (2007). Sustainable Innovation. Key conclusions from sustainable innovation conferences 2003-2006. The Centre for Sustainable Design.

Chen, Y. S. (2008). The driver of green innovation and green image-green core competence. Journal of Business Ethics, 81(3), 531-543.

Churchill, N. \& Lewis, V. (1983). The five stages of small business growth. Harvard Business Review, 61(3), 30-50.

Creswell, J. (2005). Research Design: Qualitative, Quantitative, and Mixed Methods Approaches. Los Angeles: Sage.

Daim, T. U., Basoglu, A., Gunay, D., Yildiz, C., \& Gomez, F. (2013). Exploring technology acceptance for online food services. International Journal of Business Information Systems, 12(4), 383-403. DOI: 10.1504/IJBIS.2013.053214.

De Marchi, V., \& Grandinetti, R. (2012). Who are the green innovators? An empirical analysis of firm's level factors driving environmental innovation adoption. Copenhagen: Druid Conference.

Del Río, P. (2005). Analysing the factors influencing clean technology adoption: a study of the Spanish pulp and paper industry. Business Strategy and the Environment, 14(1), 20-37. DOI: $10.1002 /$ bse.426.

Demirel, P., \& Kesidou, E. (2011). Stimulating different types of eco-innovation in the UK: Government policies and firm motivations. Ecological Economics, 70(8), 1546-1557. DOI: 10.1016/j.ecolecon.2011.03.019.

Doran, J., \& Ryan, G. (2012). Regulation and firm perception, eco-innovation and firm performance. European Journal of Innovation Management, 15(4), 421-441. DOI: $10.1108 / 14601061211272367$.

Esty, D., \& Winston, A. (2009). Green to gold: How smart companies use environmental strategy to innovate, create value, and build competitive advantage. New York: John Wiley y Sons.

Freeman, C. (1996). The greening of technology and models of innovation. Technological Forecasting and Social Change, 53, 27-39. DOI: 10.1016/0040-1625(96)00060-1.

Fussler, C., \& James, P. (1996). Driving Eco-Innovation: A Breakthrough Discipline for Innovation and Sustainability. London: Pitman Publishing.

García, A., Sánchez, J., \& Marchante, M. (2015). Eco-innovation and management: An empirical analysis of environmental good practices and labour productivity in the Spanish hotel industry. Innovation, 17, 58-68. DOI: 10.1080/14479338.2015.1011057.

Greiner, L. (1997). Evolution and Revolution as Organizations Grow: A company's past has clues for management that are critical to future success. Family Business Review, 10(4), 397409. DOI: $10.1111 / \mathrm{j} .1741-6248.1997 .00397 . x$.

Hitchens, D., Thankappan, S., Trainor, M., Clausen, J., \& De Marchi, B. (2005). Environmental performance, competitiveness and management of small businesses in Europe. Royal Dutch Geographical Society, 96(5), 541-557. DOI: 10.1111/j.1467-9663.2005.00485.x.

Hunter, C., \& Shaw, J. (2007). The ecological footprint as a key indicator of sustainable tourism. Tourism Management, 28(1), 46-57. DOI: 10.1016/j.tourman.2005.07.016.

Hojnik, J., \& Ruzzier, M. (2016). What drives eco-innovation? A review of an emerging literature. Environmental Innovation and Societal Transitions, 19, 31-41. DOI: 10.1016/j. eist.2015.09.006.

Hong, C., \& Shuai, S. (2008). Research of local government behavior in eco-technological innovation process. Management Science and Engineering, 2(1), 86-96. DOI: 968/j. mse.1913035X20080201.011.

Horbach, J., Rammer, C., \& Rennings, K. (2012). Determinants of eco-innovations by type of environmental impact. The role of regulatory push/pull, technology push and market pull. Ecological Economics, (78), 112-122. DOI: 10.1016/j.ecolecon.2012.04.005. 
Horng, J. S., Chou, S., Liu, C., \& Tsai, C. (2013). Creativity, aesthetics and eco-friendliness: A physical dining environment design synthetic assessment model of innovative restaurants. Tourism Management, 36, 15-25. DOI: 10.1016/j.tourman.2012.11.002.

Horng, J. S., Wang, C., Liu, C., Chou, S., \& Tsai, C. (2016). The Role of Sustainable Service Innovation in Crafting the Vision of the Hospitality Industry. Sustainability, 8(3), 1-18. DOI: $10.3390 /$ su8030223.

Hsiao, T. Y., \& Chuang, C. (2016). Creating shared value through implementing green practices for star hotels. Asia Pacific Journal of Tourism Research, 21(6), 678-696. DOI: 10.1080/10941665.2015.1068194.

Jänicke, M. (2008). Ecological modernisation: new perspectives. Journal of Cleaner Production, 16(5), 557-565. DOI: 10.1016/j.jclepro.2007.02.011.

Johnston, W., Leach, M., \& Liu, A. (1999). Theory testing using case studies in business-to-business research. Industrial Marketing Management, 28, 201-213. DOI: 10.1016/S00198501(98)00040-6.

Karakaya, E., Hidalgo, A., \& Nuur, C. (2014). Diffusion of eco-innovations: A review. Renewable and Sustainable Energy Reviews, 33, 392-399. DOI: 10.1016/j.rser.2014.01.083.

Kemp, R. (2010). Eco-Innovation: definition, measurement and open research issues. Economía Política, 27(3), 397-420. DOI: 10.1428/33131.

Kemp, R., \& Arundel, A. (1998). Survey indicators for Environmental Innovation. Oslo: IDEA report. Step group.

Kemp, R., \& Horbach, J. (2007). Measurement of competitiveness of eco-innovation. Measuring Eco-innovation Project (MEI).

Klemmer, P., Lehr, U., \& Lobbe, K. (1999). Innovation effects of environmental policy instruments. Berlin: Analytica-Verlag.

Klewitz, J., \& Hansen, E. (2014). Sustainability-oriented innovation of SMEs: a systematic review. Journal of Cleaner Production, 65, 57-75. DOI: 10.1016/j.jclepro.2013.07.017.

Lazaric, N., \& Dennis, B. (2005). Routinisation and memorisation of tasks inside a workshop: an illustration through a case study. Industrial and Corporate Change, 14(5), 873-896. DOI: 10.1093/icc/dth074.

Little, A. D. (2005). How leading companies are using sustainability-driven innovation to win tomorrow's customers. Innovation High Ground Report.

Molero, J., \& García, A. (2008). The innovative activity of foreign subsidiaries in the Spanish Innovation System: An evaluation of their impact from a sectoral taxonomy approach. Technovation (28), 739-757. DOI: 10.1016/j.technovation.2008.03.005.

Mossalanejad, A. (2011). The role of economic policy and environment in sustainable development. International Journal of Environmental Research, 5(2), 395-402.

Oltra, V., \& Saint Jean, M. (2009). Sectoral systems of environmental innovation: an application to the French automotive industry. Technological Forecasting and Social Change, 76, 567583. DOI: $10.1016 /$ j.techfore.2008.03.025.

Peiró, A., Segarra, M., \& Verma, R. (2014). The impact of environmental certification on hotel guest ratings. Cornell Hospitality Quarterly, 55(1), 40-51. DOI: 10.1177/1938965513503488.

Perramon, J., Alonso, M., \& Llach, J. (2014). Green practices in restaurants: Impact on firm performance. Operations Management Research, 7(1-2), 2-12.

Przychodzen, J., \& Przychodzen, W. (2015). Relationships between eco-innovation and financial performance-evidence from publicly traded companies in Poland and Hungary. Journal of Cleaner Production(90), 253-263. DOI: 10.1016/j.jclepro.2014.11.034.

Rennings, K. (2000). Redefining innovation - eco-innovation research and the contribution from ecological economics. Ecological Economics, 32(2), 319-332. DOI: 10.1016/S09218009(99)00112-3.

Rennings, K., \& Zwick, T. (2003). Employment Impacts of Cleaner Production (Vol. 21). Heidelberg: ZEW Economic Studies. 
Rodgers, S. (2007). Innovation in food service technology and its strategic role. International Journal of Hospitality Management, 26(4), 899-912. DOI: 10.1016/j.ijhm.2006.10.001.

Rosen, C. M. (2001). Environmental strategy and competitive advantage: an introduction. California Management Review, 43(3), 8-10.

Ruiz, C. C., Pérez, E., \& Fenech, F. (2013). La sostenibilidad y el papel de la contabilidad en la gestión del cambio climático y la ecoinnovación en la pyme [Sustainability and the role of accounting in the management of climate change and eco-innovation in SMEs]. Cuadernos Económicos de ICE, 86, 53-76.

Segarra, M. D., Peiró, A., Miret, L., \& Albors, J. (2011). ¿Eco-innovación, una evolución de la innovación? Análisis empírico en la industria cerámica española [Eco-innovation, an evolution of innovation? Empirical analysis in the Spanish ceramic industry]. Boletín de la Sociedad Española de Cerámica y Vidrio, 50(5), 253-260. DOI: doi:10.3989/cyv.332011.

Segarra, M. D., Peiró, A., Mondéjar, J., \& Vargas, M. (2014). Service vs. manufacturing: how to address more effectively eco-innovation public policies by disentangling the different characteristics of industries. Innovation: The European Journal of Social Science Research, 27(2), 134-151. DOI: 10.1080/13511610.2013.863705.

Tarí, J. J., Claver, E., Pereira, J., \& Molina, J. (2010). Levels of quality and environmental management in the hotel industry: Their joint influence on firm performance. International Journal of Hospitality Management, 29(3), 500-510. DOI: 10.1016/j.ijhm.2009.10.029.

Teng, C. C., \& Chang, J. (2014). Effects of temporal distance and related strategies on enhancing customer participation intention for hotel eco-friendly programs. International Journal of Hospitality Management, 40, 92-99. DOI: 10.1016/j.ijhm.2014.03.012.

Tesone, D. V. (2004). Development of a sustainable tourism hospitality human resources management module: a template for teaching sustainability across the curriculum. International Journal of Hospitality Management, 23(3), 207-237. DOI: 10.1016/j.ijhm.2003.10.003.

Tzschentke, N. A., Kirk, D., \& Lynch, P. (2008). Going green: Decisional factors in small hospitality operations. International Journal of Hospitality Management, 27(1), 126-133. DOI: 10.1016/j.ijhm.2007.07.010.

Van Berkel, R. (2007). Eco-Innovation: opportunities for advancing waste prevention. International Journal of Environmental Technology and Management, 7(5-6), 527-550. DOI: 10.1504/IJETM.2007.015629.

Velázquez, J., \& Vargas, E. (2015). De la innovación a la ecoinnovación. Gestión de servicios en empresas hoteleras [From innovation to eco-innovation. Service management in hotel companies]. Revista Venezolana de Gerencia, 20(70), 267-281.

Yin, R. (2009). Case study research. Design and methods (4a ed.). London: Sage Publications. 